e-migrinter

e-Migrinter

$22 \mid 2021$

Varia

\title{
Therrien, Catherine (2019) Celui qui échoue devient
} sorcier

Jordan Pinel

\section{(2) OpenEdition}

Journals

Édition électronique

URL : https://journals.openedition.org/e-migrinter/2790

DOI : 10.4000/e-migrinter.2790

ISSN : 1961-9685

Éditeur

UMR 7301 - Migrinter

Référence électronique

Jordan Pinel, «Therrien, Catherine (2019) Celui qui échoue devient sorcier », e-Migrinter [En ligne], 22 I

2021, mis en ligne le 25 novembre 2021, consulté le 17 décembre 2021. URL : http://

journals.openedition.org/e-migrinter/2790; DOI : https://doi.org/10.4000/e-migrinter.2790

Ce document a été généré automatiquement le 17 décembre 2021.

Tous droits réservés 


\title{
Therrien, Catherine (2019) Celui qui échoue devient sorcier
}

\author{
Jordan Pinel
}

\section{RÉFÉRENCE}

Therrien, Catherine (2019) Celui qui échoue devient sorcier. Parcours d'un migrant camerounais parti d'Afrique et arrivé... en Afrique. Laval, Presses Universitaires de Laval, $252 \mathrm{p}$.

1 Dans cet ouvrage, Catherine Therrien, anthropologue à l'Université Al Akhawayn (Maroc) nous transmet l'histoire de Jackson Abena Banyomo, «migrant camerounais parti d'Afrique et arrivé... en Afrique ». Ce livre n'est pas un ouvrage scientifique et ne se présente pas comme tel. Il livre le récit de Jackson sous la forme d'une retranscription de son parcours migratoire. Il s'agit donc surtout d'un dialogue, avec des interventions courtes de l'auteure qui pose des questions de précision ou des relances pour comprendre le récit livré par Jackson. Il en ressort donc une version très orale qui - bien que rendant parfois le discours et les évènements qui se succèdent difficiles à suivre - permet de garder l'authenticité de l'échange et surtout des propos de l'enquêté, tout en apportant un certain dynamisme et une vivacité dans la lecture. Livrer une retranscription dans sa forme brute est assez inhabituel en sciences sociales, notamment car l'ouvrage laisse peu de place à l'interprétation de l'anthropologue et la contextualisation de son travail. En effet, cette dernière, après avoir expliqué sa démarche en début d'ouvrage, a seulement compartimenté le recueil de la parole de Jackson en y ajoutant des titres de partie résumant son propos. Ainsi, il ne faut pas chercher dans ce livre une analyse anthropologique ou voir dans le récit un témoignage représentatif des parcours migratoires similaires, mais bien le simple recueil d'une parole qui ne tombe pas dans le pathos.

2 Une fois passée la «surprise » que peut être un tel ouvrage pour des chercheurs en sciences sociales habitués à lire des analyses d'enquêtes entrecoupées de passages 
d'entretien, il ne reste qu'à découvrir la vie de Jackson telle qu'il nous la transmet. Le récit débute au Cameroun où Jackson passe son enfance en alternant ses premiers jobs, se marie et élève ses trois enfants, tout en rêvant « d'une vie meilleure ». Cette dernière se concrétise dans ce qu'il appelle une «envie d'Europe " qui le conduit à mettre en place son départ du Cameroun le 5 mai 2013, avec sa cousine. Son récit reprend ce que les différentes études sur les parcours migratoires au Sahel, dans le Sahara ou encore au Maghreb ont mis en avant: le voyage, long et souvent pénible, les combines et les envois d'argent depuis le pays d'origine par un proche, les passeurs, les tentatives pour passer en Europe. La peur aussi, souvent. La peur pour lui, sa vie, mais aussi pour celle de sa cousine - «sa sœur » comme il la surnomme. Le parcours de Jackson l'a conduit au Nigéria, au Niger, en Algérie, puis au Maroc, par de multiples arrêts plus ou moins longs, répertoriés sur une carte au début de son récit. Le lecteur peut ainsi suivre son périple à rebours et avec des retours en arrière régulier : son départ du Cameroun, le passage de la frontière entre le Nigéria et le Niger, le bus entre Tamanrasset (Algérie) et Oran (Algérie), l'arrêt à Béchar (Algérie) pour travailler pendant que « sa sœur » restait à Oran dans un nganda, la traversée vers le Maroc puis le refoulement vers l'Algérie, la deuxième traversée et l'arrivée à Oujda (Maroc).

Arrivé à son premier tiers, le récit se concentre alors principalement sur la période de vie de Jackson au Maroc. D'abord, son objectif est de se rendre à Tanger où il souhaite parvenir en Europe par la mer. À plusieurs reprises - son histoire suivant sa mémoire et des moments de "parenthèses " dans le récit - nous sommes perdus dans l'ordre chronologique des évènements. C'est là où le rôle pivot de l'anthropologue s'exerce afin de "raccrocher" les morceaux par ses questions et de mieux comprendre ce que Jackson tente d'expliquer: d'abord ses tentatives infructueuses pour gagner l'Espagne depuis Tanger en zodiac, puis la séparation d'avec sa cousine qui restera à Tanger pour finalement être reconnue réfugiée par le Haut-Commissariat des Nations unies pour les réfugiés (HCR), tandis que Jackson tentait sa chance du côté de Nador (Maroc). Ou plutôt, dans la forêt de Gourougou près de Nador, à cinq kilomètres de la frontière le séparant de Melilla (Espagne), enclave européenne la plus proche de lui à ce moment ce qui lui donnait la « rage d'entrer en Europe » (p.102). Là, pendant huit mois, la vie de Jackson s'est partagée entre la survie dans la forêt avec plus de 700 autres personnes dans des conditions inhumaines de clandestinité, de proximité et d'insalubrité et entre ses nombreuses tentatives de franchissement de la barrière érigée entre le Maroc et l'Espagne. Le démantèlement du camp l'obligera à se rendre à Tiznit, tout au sud du Maroc, dans un camp organisé par l'État. Puis, il se rend à Meknès en 2015 où il espère toujours pouvoir rejoindre l'Europe un jour. C'est là où son projet a pris un tout autre chemin. Accueilli dans une église de Meknès, il fait la rencontre du Père Jean, investi dans l'association Caritas qui vient notamment en aide aux migrants. Jackson, fatigué, décide de rester dans la ville et de s'investir bénévolement dans l'association. Il vient notamment en aide aux migrants subsahariens qui vivent dans le " ghetto » de Meknès, décrivant la violence subie par nombre d'entre eux, la prostitution de certaines femmes, et laissant ainsi entrevoir au lecteur d'autres exemples de conditions de vie difficiles des migrants au Maroc.

4 C'est à Meknès que Jackson prend la décision de rester au Maroc et qu'il cherche à se faire régulariser, notamment grâce à une campagne de régularisation marocaine. Le 17 octobre 2017, plus de quatre ans après son départ du Cameroun, il obtient sa carte de séjour, ce qui le soulage et le rend fier après tout le parcours entrepris : «je n'ai plus rien ressenti comme fatigue. J'étais TELLEMENT fier de moi...»(p.169). Après avoir 
obtenu un appartement, un emploi avec Caritas, Jackson peut enfin « se poser ». C'est dans la tranquillité de cette situation qu'il peut aujourd'hui revenir sur son parcours avec un certain recul. Il revient notamment sur l'émergence des projets migratoires et sur les «mensonges» relayés par beaucoup de migrants ayant échoué selon lui dans leur entreprise migratoire. C'est à ce moment que le titre de l'ouvrage, encore obscur, devient clair : «Au Cameroun, on a un adage qui dit : « quand tu échoues dans la vie, tu deviens un grand sorcier ». Pourquoi? Parce que quand tu rates ta vie, tu veux que tout le monde soit comme toi. Tu cherches à attirer un maximum de personnes dans ta situation pour ne pas être le seul à avoir honte.» (p.199). Jackson veut aujourd'hui se positionner en opposition à cette posture du sorcier en aidant les migrants subsahariens au Maroc en leur montrant la réalité du parcours qui les attend et la difficulté qui existe encore en Europe. Il se positionne ainsi, d'autant plus que lui, resté au Maroc et régularisé, peut aujourd'hui se rendre en France pour des déplacements dans le cadre de son travail et de sa formation.

On peut noter un manque dans ce récit: l'absence de critique vis-à-vis des politiques migratoires, notamment européennes, et de l'externalisation des frontières qui rend le parcours si difficile. Lorsque Jackson évoque les « sorciers » comme en partie à l'origine du départ d'autres migrants, son discours semble vouloir dire que le départ vers l'Europe n'est pas une solution, au contraire elle est source de grandes difficultés dans la plupart des cas. Mais, le lecteur doit alors se rappeler que ces difficultés sont surtout causées par la fermeture des frontières et l'externalisation des frontières. L'ouvrage manque alors peut être de références scientifiques qui pourraient accompagner le lecteur sur les réalités migratoires derrière le récit de Jackson. Mais ce n'est pas le but affiché par Catherine Therrien, qui souhaite clairement laisser la parole à l'enquêté et à sa vision du parcours. Et c'est un récit honnête que fait Jackson de son parcours, ses réactions face aux dangers, à la peur, à la lassitude, au message qu'il souhaite transmettre aux autres migrants et aux futurs migrants. Il évoque ainsi le moment où il est parti au commissariat pour se livrer aux policiers marocains, las de vivre de manière inhumaine dans cette forêt. Il évoque aussi sa relation qu'il aura quelques années plus tard avec Charlotte, une française au Maroc, dont il évoque les difficultés de compréhension au sein du couple et le fossé qui s'est creusé entre eux face aux différences de parcours. Un regard réflexif sur son parcours, son passé et qui il est devenu aujourd'hui qui rend le témoignage sensible: ce n'est pas le Jackson d'il y a quelques années qui parle, mais celui qui vit aujourd'hui au Maroc, en étant heureux, en travaillant, en aidant les autres. Son témoignage, bien que montrant toutes les difficultés auxquelles sont confrontés un grand nombre de migrants en Afrique à cause des politiques migratoires mondiales, donne un peu d'optimisme. Son accomplissement spirituel et humanitaire sur lequel il revient après avoir évoqué toutes ses difficultés, rend compte de sa ténacité. 


\section{AUTEURS}

JORDAN PINEL

Docteur en géographie

Post-doctorant à la Caisse Nationale d'Assurance Vieillesse 